\title{
MACROSCOPIC INVESTIGATION OF HYDRATE FILM GROWTH AT THE HYDROCARBON/WATER INTERFACE
}

\author{
Craig J. Taylor, Kelly T. Miller, Carolyn A. Koh, E. Dendy Sloan * \\ Department of Chemical Engineering \\ Colorado School of Mines \\ 1600 Illinois St, Golden, CO, 80401 \\ USA
}

\begin{abstract}
Hydrate film growth has been examined at the hydrocarbon/water interface for cyclopentane and methane hydrate. Video microscopy was used to measure hydrate film thickness, propagation rate across the hydrocarbon/water interface and gas consumption measurements characterized the hydrate formation mechanism. Cyclopentane and methane hydrate film formation were measured over the temperature range of $260-279 \mathrm{~K}$ and pressure range of atmospheric to $8.3 \mathrm{MPa}$. Hydrate formation was initiated by the propagation of a thin, porous film across the hydrocarbon/water interface. The propagation rate and thickening of the hydrate film was strongly dependent on the hydrate former solubility in the aqueous phase, in the absence and presence of hydrate. Cyclopentane hydrate film thickness began at $\sim 12 \mu \mathrm{m}$ and grew to a final thickness $(15-40 \mu \mathrm{m})$ which increased with subcooling. Methane hydrate film thickness began at $\sim 5 \mu \mathrm{m}$ and grew to a final thickness $(20-100 \mu \mathrm{m})$ which also increased with subcooling. The hydrate film grew into the water phase. Gas consumption measurements indicated that the aqueous phase supplied hydrate former during the initial hydrate growth, and the free gas supplied the hydrate former for film thickening and development. Hydrate film formation at the hydrocarbon/water interface was proposed to consist of three consecutive stages: propagation, development and bulk conversion.
\end{abstract}

Keywords: Clathrate hydrate; Crystallization; Hydrate Film formation; Optical microscopy.

\section{INTRODUCTION}

Clathrate hydrates are ice-like crystalline compounds which form as thin porous crystalline films at the interface between the water phase and the hydrocarbon guest molecule phase (gas or liquid) [1-3]. The growth characteristics of these hydrate films are of significant importance in seafloor $\mathrm{CO} 2$ sequestration, gas hydrate transport, and flow assurance in oil and gas pipelines.

A proposed model for hydrate formation in crude oil pipelines [4] suggests that water droplets first become entrained in the oil phase, hydrates then nucleate at the water/hydrocarbon interface to form hydrate shells around the water droplets, and finally the hydrate shell grows into the droplet interior, converting the remaining water to hydrate. However, it is unclear how much of the water droplet is converted to hydrate upon shell formation and how long it takes to fully convert the droplet to hydrate. Answering such fundamental questions and directly verifying the water droplet to hydrate shell conversion were the motivation for characterizing hydrate film growth.

The film thickness [5-11] and propagation growth rate (hydrate film growth along the hydrocarbon/ water interface) $[8,12-14]$ of hydrates has been experimentally studied for a variety of hydrate formers. The hydrate film thicknesses measured in

\footnotetext{
* Corresponding author: Phone: +1 (303) 273-3723 Fax +1 (303) 273-3730 E-mail: esloan @ mines.edu
} 
these studies ranged over three orders of magnitude, from microns to millimeters.

The large variability between past measurements has been attributed to differences among experimental techniques, thermodynamics conditions, hydrate guests, hydrate structure, age of the hydrate film, amount of water used, and the saturation of the aqueous phase [9]. Conditions such as pressure, temperature, and time can also drastically change the hydrate film thickness [8]. The hydrate guest and structure have also been shown to significantly change the hydrate film thickness, even using the same experimental technique [9]. From the present work, we hypothesize that the amount of water used and the solubility of the hydrate former in the aqueous phase also affect the hydrate growth.

Propagation rates of hydrate films have been previously studied for $\mathrm{CO} 2$ and methane hydrates. Propagation rates for methane hydrate were on the order of tens of microns per second [8, 12], whereas $\mathrm{CO} 2$ hydrate were on the order of millimeters per second [13-15]. Measurements have shown the propagation rate to be a function of temperature, pressure, and hydrate structure.

Several experimental techniques have been used to measure the hydrate film thickness and propagation rate at the hydrocarbon/water interface, including laser interferometry $[9,11]$, micrometer measurements [8], magnetic resonance imaging (MRI) [6], and visual microscopy $[7,16]$. Interferometry measurements of hydrate film thickness with the refrigerant $134 \mathrm{a}$ and water [9] showed distinct advantages over visual techniques. However, it would be extremely difficult to perform such measurements with carbon dioxide or methane hydrate films due to the high formation pressures for these gas hydrate formers.

The present study uses digital video microscopy to investigate hydrate film thickness as a function of time, as well as propagation rate for methane and cyclopentane hydrate. This study enhances past measurements of hydrate film growth by incorporating gas consumption measurements simultaneously with film thickness measurements to determine which phase supplies the hydrate former during hydrate formation. This study also advances the physical knowledge of hydrate formation by relating film formation with the water droplet to hydrate shell conversion.

\section{BACKGROUND}

Initial micromechanical studies of cyclopentane hydrate led to a technique to heterogeneously nucleate cyclopentane hydrate particles [17]. In this technique, a water droplet was placed on a cantilever and submerged in cyclopentane, before being nucleated by another hydrate particle (Figure 1A). Nucleation occurred upon contact, and a thin hydrate porous shell formed around the water droplet within a few minutes (Figure 1B). While the hydrate film encompassed the entire surface of the water droplet, the interior of the particle contained unconverted water. Over time, cyclopentane diffused through the hydrate layer, and the water inside the hydrate shell was converted into hydrate. Inward dimples then grew on the shell indicating continued growth (Figures 1C and 1D) [18]. As the interior of the droplet converts to hydrate, the particle darkens due to light scattering (Figure 1E). Finally, after seven hours the entire particle darkens due to further hydrate conversion (Figure 1F).
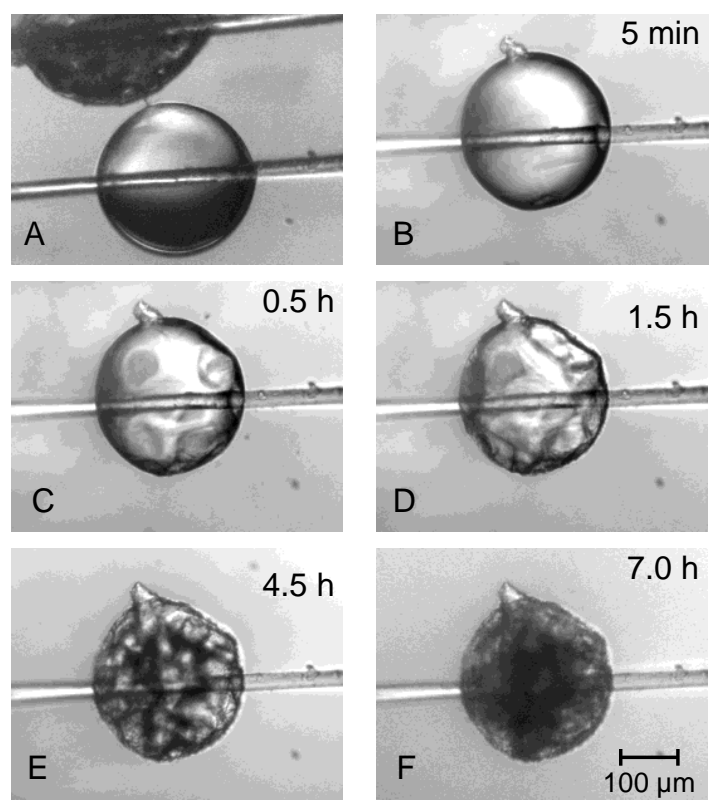

Figure 1. Nucleation of a water droplet immersed in cyclopentane. (A) Initial contact, (B) cyclopentane hydrate shell formed around the water droplet, (C) dimples formed on the hydrate shell, (D) continued dimple, (E) conversion of interior water to hydrate, indicated by darkening, (F) almost completely converted hydrate. 
Since the above cyclopentane micromechanical experiments could be only performed at atmospheric pressure, a subsequent apparatus was designed to investigate hydrate film/shell growth at a planar hydrocarbon/water interface. The film growth apparatus was used to study shell/film formation at pressures of up to $14 \mathrm{MPa}$. In addition to cyclopentane hydrate (a model sII hydrate which is stable at atmospheric pressure), methane hydrate film growth was examined. Growth of methane hydrate shells on water droplets has been suggested to be the mechanism of hydrate formation in subsea pipelines [4, 19].

\section{EXPERIMENTAL PROCEDURE}

The apparatus used to investigate interfacial hydrate film formation consisted of a brass cell of $5.5 \mathrm{~cm} 3$ interior volume housing two sapphire windows ( $1.0 \mathrm{~cm}$ diameter) which was identical to the cell used by Freer. [12]. The brass body of the cell was surrounded by a cooling jacket connected to a recirculating cooling bath (Figure 2). The cell was positioned on a vibration isolation table. A microscope (Olympus SZ60, Objective: $100 \mathrm{AL}$ $2 \mathrm{X}$ ) was used to view the gas-water interface through the sapphire window. The cell was pressurized with gas from the top of the cell, and a pressure gauge and transducer were used to monitor the pressure inside the cell. A T-type thermocouple on the outside of the brass cell recorded the temperature. The pressure and temperature were monitored continuously using a data acquisition system.

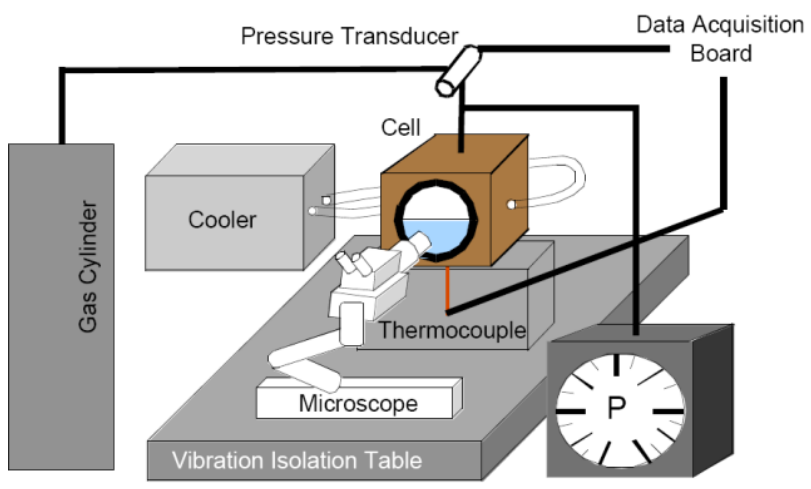

Figure 2. Schematic of the hydrate film growth apparatus.

\section{Experimental technique}

In order to investigate hydrate formation at the hydrocarbon/water interface, approximately half the cell $(2.5 \mathrm{~cm} 3)$ was filled with HPLC grade water. For cyclopentane experiments, $\sim 2.0 \mathrm{~cm} 3$ of liquid cyclopentane (95\%, Aldrich) was added to the water. For methane $(99.99 \%$, Matheson) experiments, the cell containing water was typically pressurized to $6.9 \mathrm{MPa}$ at $25{ }^{\circ} \mathrm{C}$ and allowed to equilibrate. After gas dissolution in the water, the temperature was lowered to the hydrate equilibrium temperature and allowed to equilibrate. The temperature was then lowered further to the desired subcooling. After subcooling had been achieved and dissolution completed, the pressure remained constant for several minutes until hydrate nucleation occurred. During the entire process, the temperature and pressure were recorded to determine the number of moles of gas consumed during dissolution and hydrate formation.

\section{Image acquisition and analysis}

Time lapsed videos of the film growth and thickening process were recorded using a greyscale 1/2" CCD camera (Cohu, Model 49152030) and directly digitized using a framegrabber card (Scion, LG3) in a personal computer. Image processing and analysis were performed using the image analysis program, ImageJ [20].

The magnification of the microscope was calibrated using a stage micrometer; each pixel in the digitized image had a length of $1.59 \mu \mathrm{m}$. The positions of the hydrate-hydrocarbon and hydratewater interfaces were located with subpixel accuracy by a calibrated interpolation technique. The apparent width of the interface, measured by the change in grey level in the acquired images, was typically 2 to 3 pixels $(3-5 \mu \mathrm{m})$. To calibrate the exact positions of the interfaces, the apparent width of a glass fiber of known diameter was measured under similar illumination conditions, and the grey level corresponding to the actual diameter was determined. This calibrated grey level was then used to determine the interface positions, by interpolating between the measured grey levels across the phase boundary.

\section{RESULTS}

The hydrate film thickness and propagation rate, along the hydrocarbon/water interface, were measured for cyclopentane and methane hydrate. The experiments were designed to characterize the 
effect of temperature, pressure, and solubility of hydrate guest on hydrate film growth.

\section{Cyclopentane Hydrate Film Growth}

The first hydrate film growth experiments were performed on cyclopentane hydrate to verify whether a hydrate shell/film, similar to that seen in the micromechanical measurements (Figure 1), was observed at the planar cyclopentane/water interface. Figure 3 shows a sequence of video images recorded during cyclopentane film growth at $3.8{ }^{\circ} \mathrm{C}$ subcooling. All phase equilibrium predictions were performed using CSMGem [21], an in-house hydrate equilibrium program. In Figure 3A, cyclopentane liquid is above the interface, and water is below. Small accumulations of hydrate began to build-up near the interface (Figure 3B), and continued to grow in Figure 3C. The hydrate film spread over the entire interface as shown in Figures 3C and 3D with a thickness of approximately $12 \mu \mathrm{m}$, which acted as a barrier between cyclopentane and water [22, 23].

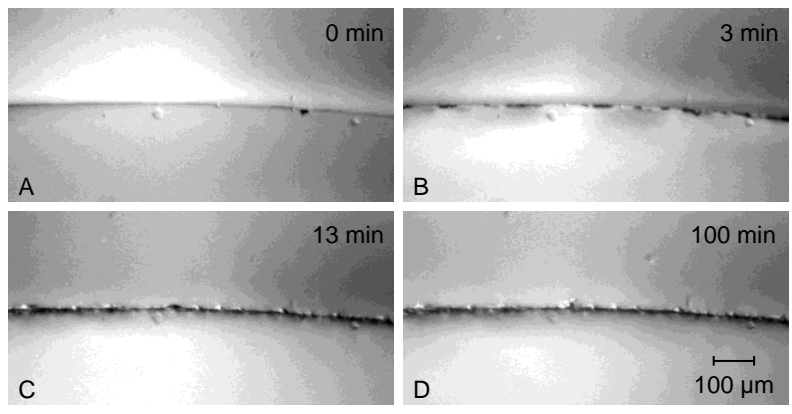

Figure 3. Cyclopentane hydrate film growth at a cyclopentane/water interface. The sequence of images was recorded over 100 minutes. Temperature $=3.2{ }^{\circ} \mathrm{C}$. Subcooling $=3.8^{\circ} \mathrm{C}$. [21] The final hydrate film thickness was $15.2 \pm 1.59$ $\mu \mathrm{m}$.

Over time, the hydrate layer was observed to thicken. Hydrate film thickness was measured during the entire hydrate formation process. Due to the ostensible film thickness variability across the interface (Figure 3D), the thickness was measured at 40 locations across the interface, and an average thickness was calculated.

Figure 4 shows the hydrate film thickness as a function of time during hydrate formation for three different subcoolings. The hydrate film had an initial thickness of $\sim 12 \mu \mathrm{m}$ and over the course of two hours grew to its final thickness. The time required to achieve the final thickness was on the order of 200 minutes for all the cyclopentane hydrate film thickness measurements. Figure 5 shows that the final hydrate film thickness increased approximately linearly between 4 to 10 ${ }^{\circ} \mathrm{C}$ subcooling.

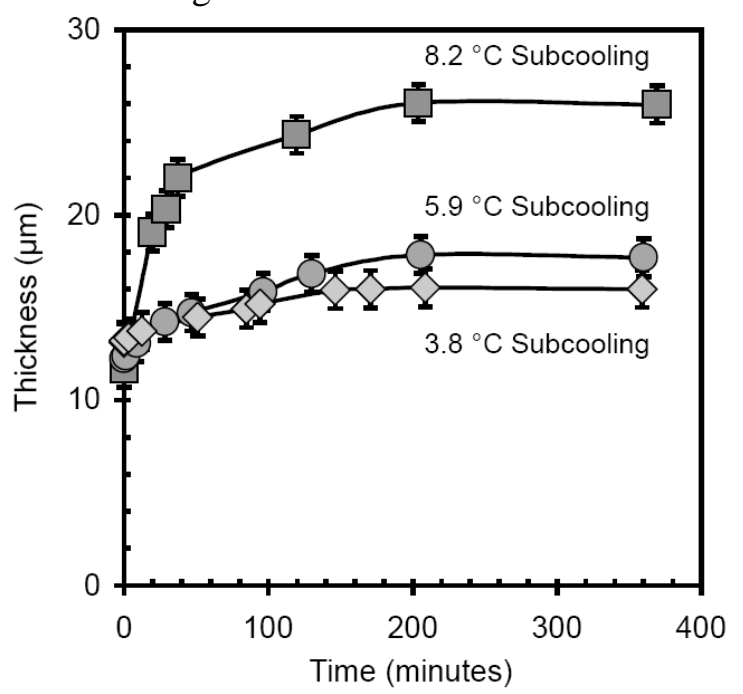

Figure 4. Cyclopentane hydrate film thickness versus time, for various subcoolings. Error bars represent one standard deviation.

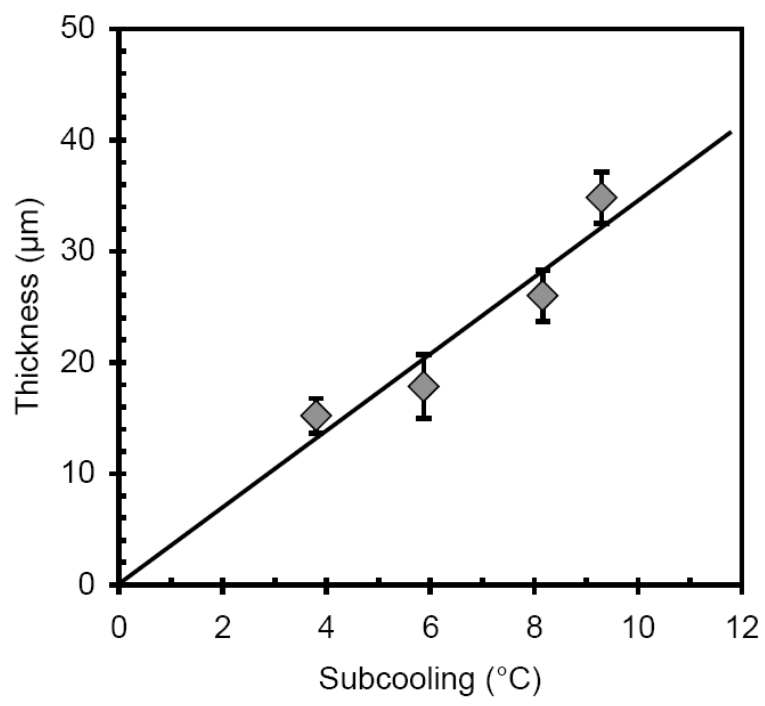

Figure 5. Final cyclopentane hydrate film thickness as a function of subcooling. Error bars represent one standard deviation.

\section{Methane Hydrate Film Growth}

After the initial experiments on cyclopentane hydrate, similar measurements were performed for methane hydrate. Figure 6 shows the progression 
of methane hydrate film growth at $6.89 \mathrm{MPa}$ and $7.45{ }^{\circ} \mathrm{C}$ subcooling [21]. Figure 6A shows the methane/water interface, where methane gas is above the interface and water is below. The initial hydrate film grew across the methane/water interface, with a thickness of $6.0 \pm 0.7 \mu \mathrm{m}$. In this particular experiment, the hydrate film propagated across the methane/water interface at a rate of 283 $\mu \mathrm{m} / \mathrm{second}$. This growth rate compared well to Freer's growth rate of $289 \mu \mathrm{m} / \mathrm{second}$ at $6.31 \mathrm{MPa}$ and $6.7^{\circ} \mathrm{C}$ subcooling [12].
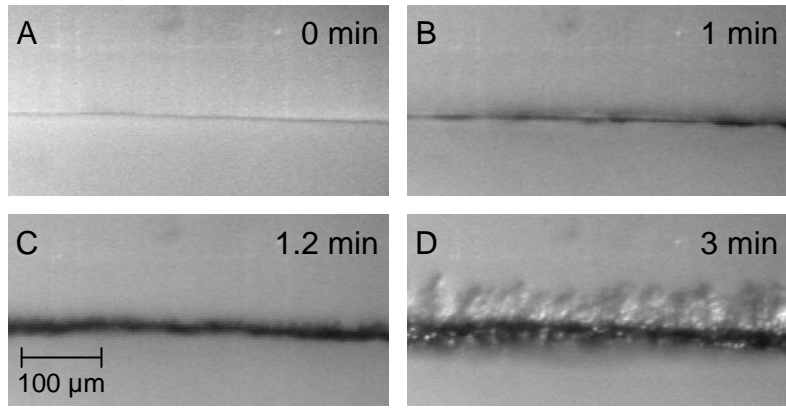

Figure 6. Methane hydrate film growth at the methane/water interface. Video images A to D were recorded over 3 minutes. Final long-term hydrate film thickness $=53.0 \pm 5.0 \mu \mathrm{m}$.

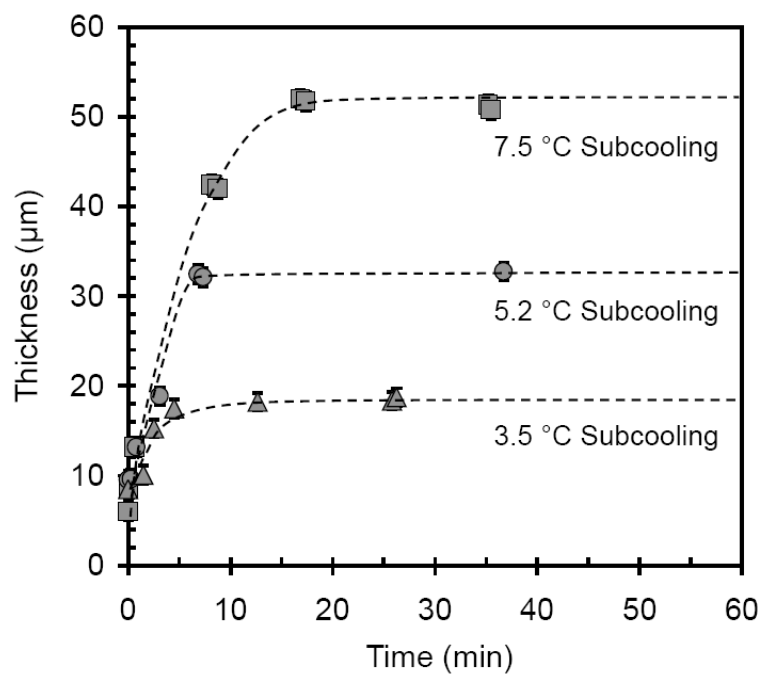

Figure 7. Methane hydrate film thickness as a function of time for various subcoolings. Error bars represent one standard deviation.

Over time, the hydrate layer was observed to thicken, presumably by diffusion of methane through the hydrate layer [2]. Figure 6B\&C show that the hydrate film grew in thickness over the course of half a minute; the hydrate film reached a final thickness of $53 \mu \mathrm{m}$ over the course of 17 minutes. Hydrate film thickness was measured throughout the entire hydrate formation process. Figure 7 shows the methane hydrate film thickness asymptotically increases over time for three different subcoolings. It should be noted that hydrate growth also occurred on the sapphire window, as indicated by the clear crystals above the dark hydrate layer in Figure 6D. With this visual technique, it is not clear whether any surface or wall effects which would alter the measured film thickness.

All three experiments had an initial methane hydrate film thickness of approximately $5 \mu \mathrm{m}$ and grew to a final thickness which depended on subcooling. In all the methane hydrate film measurements the final thickness was achieved in less than 20 minutes after nucleation, which was significantly faster than that for cyclopentane hydrate (approximately 200 minutes). This is believed to be due to the smaller molecular size of methane (4.36 $\AA)$ compared to cyclopentane $\left(\begin{array}{ll}\sim 6.40 \AA & \AA\end{array}\right)$ and therefore a larger diffusion coefficient through the hydrate layer [1].

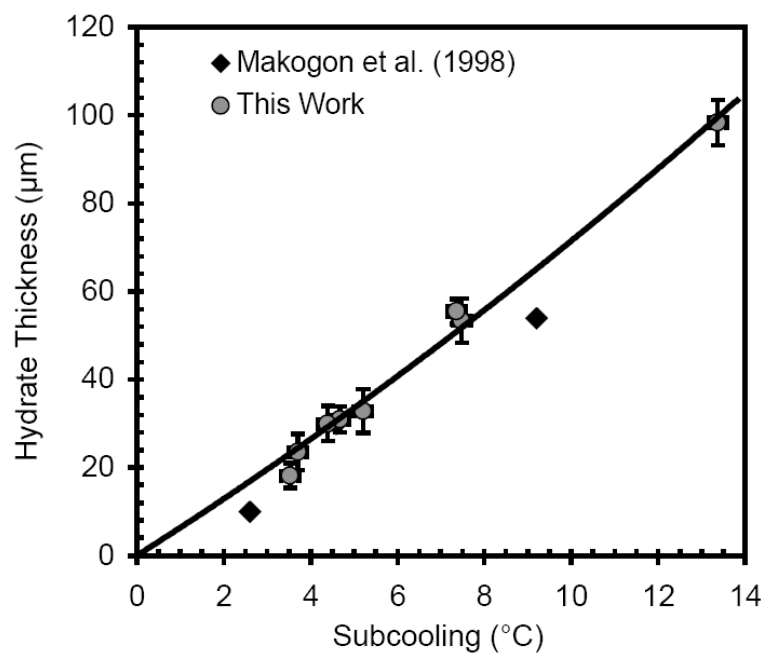

Figure 8. Final methane hydrate film thickness as a function of subcooling.

Methane hydrate film thickness measurements were performed for subcoolings between 3 to 14 ${ }^{\circ} \mathrm{C}$ (Figure 8). Similar to cyclopentane hydrate, the methane hydrate film thickness was directly proportional to subcooling. The data from the present study are in good agreement with data measured by Y. Makogon (Figure 8), who 
measured methane hydrate film thickness via a micrometer [8].

\section{Film Growth in an Oil / Methane / H2O System} In an additional experiment, $1 \mathrm{~cm} 3$ of n-decane was added to $2.5 \mathrm{~cm} 3$ water before pressurizing with methane, in order to represent a three phase (oil/methane/water) system. Adding n-decane had no effect on the film thickness. The methane hydrate film thickness measured for this threephase system was $31.0 \pm 2.9 \mu \mathrm{m}$ at $4.7{ }^{\circ} \mathrm{C}$ subcooling, which was in agreement with the value measured for methane hydrate without ndecane at a similar subcooling (Figure 8). Figure 9 compares the hydrate film thickness as a function of time for methane, methane/n-decane, and cyclopentane systems. The time required to reach $95 \%$ of the final thickness is indicated by an arrow on Figure 9 for each experiment.

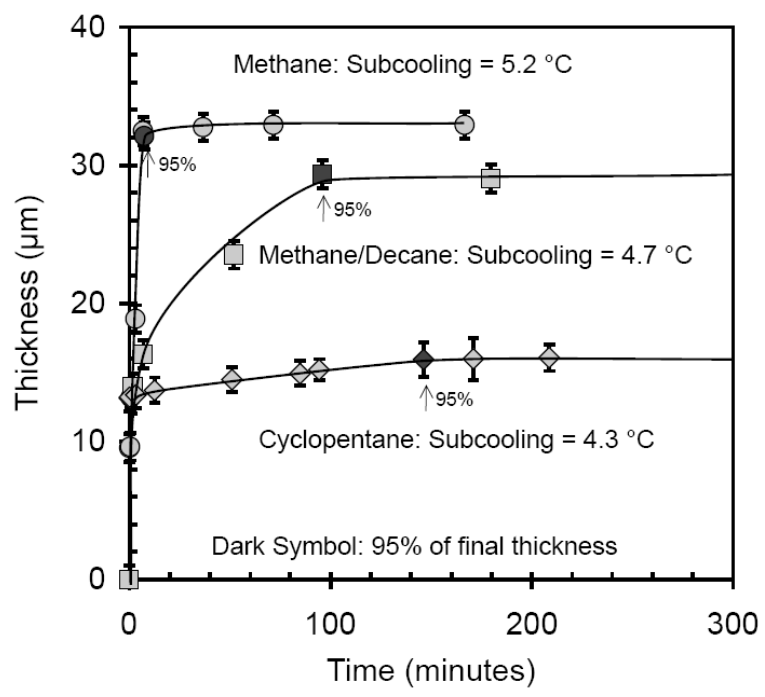

Figure 9. Hydrate film thickness versus time for the methane, methane/n-decane, and cyclopentane systems. Error bars represent one standard deviation. Time zero was recorded at the first sign of film formation.

The time to achieve the final film thickness was significantly longer for the case with n-decane present compared to the system with only methane present (Figure 9). With the addition of n-decane, nearly 100 minutes was required to produce the final thickness, compared to 20 minutes for all methane hydrate experiments without n-decane present. However, the cyclopentane hydrate film developed at an even slower rate than that for either the methane or methane/n-decane systems.
Multiple parameters which may be responsible for the different film development rates for the three systems (methane, methane/n-decane, and cyclopentane) are guest molecule size, diffusivity, solubility, hydrate structure and pressure.

\section{Film Growth/Development into Water Phase}

In all experiments the hydrate film was observed to thicken into the aqueous phase. Profiles of image brightness across the hydrate film were taken at various times throughout the hydrate formation process. Figure 10 shows the profile across the hydrate film measured at a subcooling of $5.2{ }^{\circ} \mathrm{C}$ (see Figure 7). The left side of Figure 10 (position $0-69 \mu \mathrm{m}$ ) represents the vapor side of the interface, while the right side (position 120$180 \mu \mathrm{m})$ represents the water side of the interface. The large peak in the middle indicates the hydrate phase.

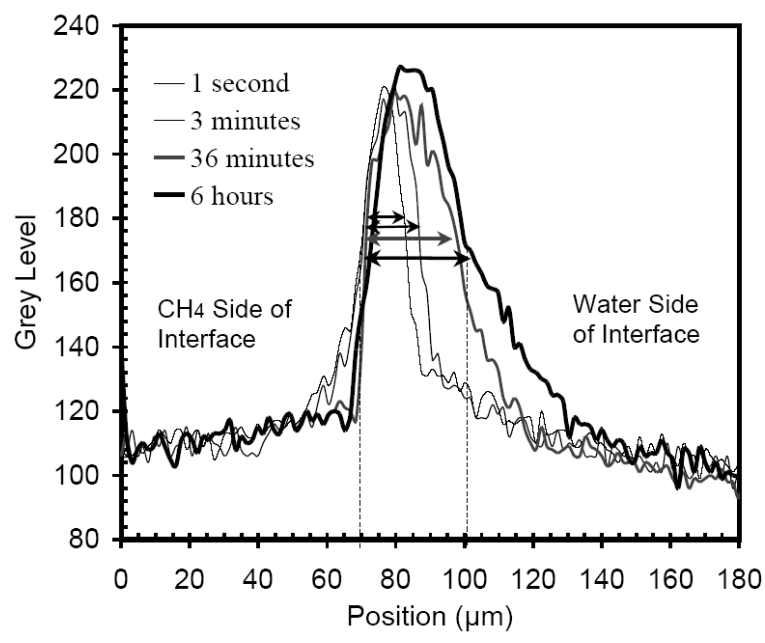

Figure 10. Profile of vapor/hydrate/water interface. The left side of graph represents top half of vapor/water interface (vapor phase) of Figure 6 and right side represents bottom half of vapor/water interface (water phase).

Immediately after the hydrate initially propagated across the interface, the hydrate film was located between positions 70 and $80 \mu \mathrm{m}$, corresponding to a $10 \mu \mathrm{m}$ hydrate film thickness. Over time, the hydrate film was observed to thicken. After 6 hours, the hydrate film was located between positions 71 and $103 \mu \mathrm{m}$, corresponding to a 32 $\mu \mathrm{m}$ film thickness. Since the methane/hydrate edge only moved from position 71 to $72 \mu \mathrm{m}$, whereas the water/hydrate edge moved from position 80 to $103 \mu \mathrm{m}$, the hydrate phase grew into 
the water phase. All of the profiles were measured to grow into the water phase. Additional profiles were taken; however, only four were plotted in Figure 10, for clarity and to illustrate the trend.

\section{Gas Consumption for Hydrate Film Growth}

During methane hydrate film growth, gas consumption data were collected to determine the amount of hydrate being formed, and to establish whether methane was supplied to the hydrate from the vapor or the aqueous phase. Figure 11 shows the methane pressure in the cell throughout an entire film growth experiment. A pressure drop indicated gas was being consumed either by aqueous phase dissolution or by hydrate formation.

The initial pressure drop from 6.63 to $6.52 \mathrm{MPa}$ in the first two hours was due to dissolution of methane into the aqueous phase and a decrease in temperature to the desired subcooling (Figure 11). After the initial two hours, the pressure remained constant for 0.5 hours. At 2 hours and 32 minutes into the experiment, hydrate film growth began. Nucleation was assumed to occur just prior to hydrate film growth (indicated by the vertical dashed line in Figure 11). After the initial hydrate film growth, the pressure decreased over the course of 22 hours as further hydrate formation occurred, indicating that methane from the vapor phase was providing a source of methane to the hydrate phase.

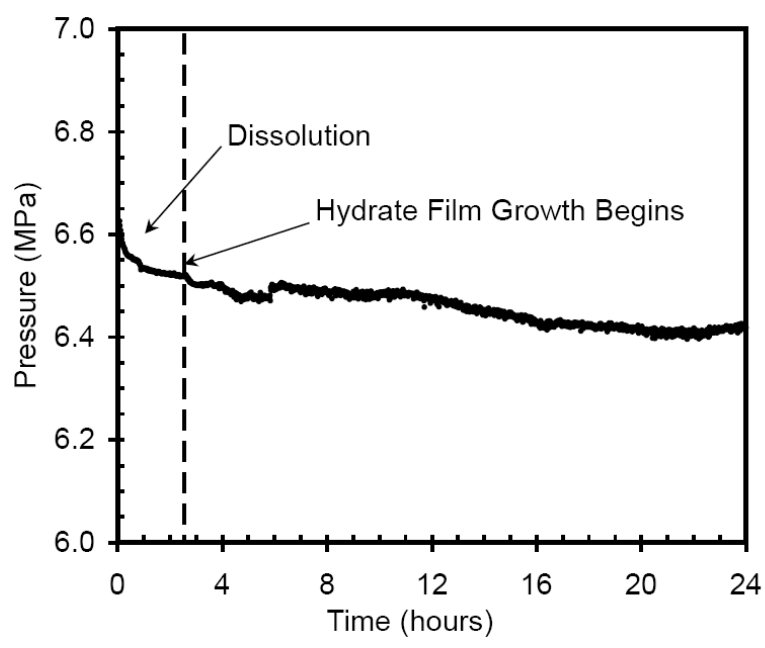

Figure 11. Cell pressure during dissolution and hydrate formation. Dissolution occurs in the first two hours. At 2 hours 32 minutes, initiation of film growth occurs, and hydrate formation causes the pressure to decrease over the next 22 hours.
However, on closer inspection of the pressure data at the beginning of hydrate film growth, no immediate decrease in pressure was observed in the first few minutes of hydrate formation (Figure 12). Such a lapse in time between the initial hydrate film growth and pressure drop was observed in all methane gas consumption experiments.

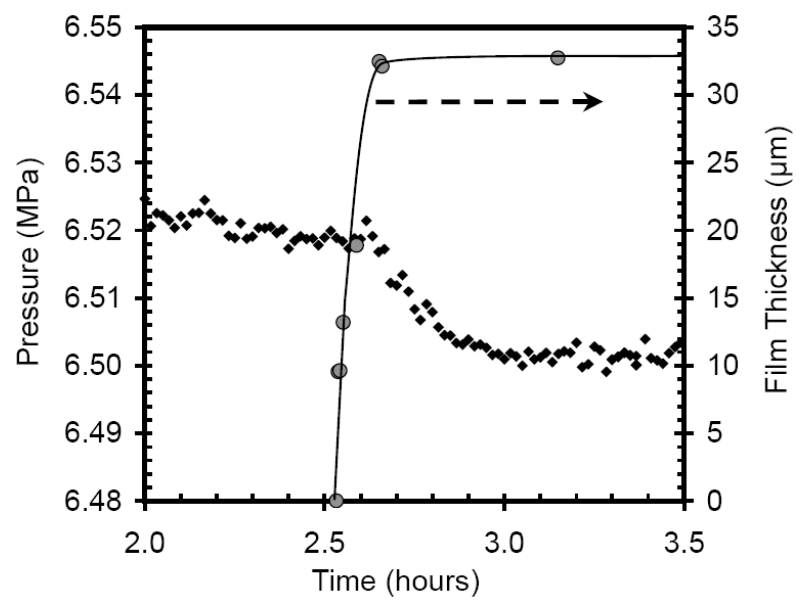

Figure 12. Cell pressure at the initiation of hydrate film growth. The pressure remained fairly constant (6.52 $\mathrm{MPa})$ for 30 minutes $(2-2.5$ hours). Initiation of film growth was visually observed at 2 hours 32 minutes. The pressure remained constant for another 5 minutes after the initiation of hydrate film growth before decreasing to 6.5 $\mathrm{MPa}$.

\section{DISCUSSION}

\section{Source of Methane for Hydrate Formation}

The absence of a pressure drop upon nucleation was observed previously in our laboratory by Subramanian and Sloan [24]. Their pressure trace lacked any discontinuities or changes in slope which would be expected upon hydrate formation in their closed vapor-liquid-water system. In order to explain the mechanism of film growth, it was necessary to know the source of methane molecules contributing to hydrate growth.

Methane molecules required for hydrate formation could be supplied from the free gas phase and/or the aqueous phase. Methane supplied from the gas would result in a decrease in pressure upon nucleation and growth of the hydrate film. However, an aqueous supply of methane would decrease the concentration of methane in the 
aqueous phase without decreasing the gas pressure upon hydrate nucleation. Since no immediate pressure drop was observed on the initiation of hydrate formation, methane may be initially supplied by the aqueous phase [24]. As the hydrate film grew across the vapor/liquid interface, a supply of concentrated methane molecules was needed near the vapor/liquid interface to preserve hydrate film growth. A concentration driving force of methane molecules was provided by the decrease in solubility of methane in water in the presence of a hydrate phase.

\section{Solubility of Methane in Water}

The methane solubility in water in the absence and presence of hydrates at $6.5 \mathrm{MPa}$ as calculated by CSMGem [21] is shown in Figure 13. This calculation is in excellent agreement with measured methane solubility data reported by Handa et al. [25]. The metastable methane solubilities in the absence of hydrates (dotted line) increase with decreasing temperature. However, with a hydrate phase present (solid line), the trend was reversed. In the absence of hydrate, the metastable methane concentration increased as the temperature was decreased below the equilibrium temperature to Point A (along the dotted line). When hydrate formation occurred, the solubility of methane in the aqueous phase decreases as equilibrium was established with the hydrate phase (Point B). After hydrate formation, the aqueous phase became supersaturated with methane as the hydrate film starts to form. As dissolved methane was consumed by hydrate film growth, the concentration of methane near the interface becomes depleted in methane relative to the bulk solution. This concentration gradient caused mass transfer of dissolved methane from the bulk aqueous phase to the bottom of the hydrate film [24].

Tohidi et al. [22, 23] used glass micromodel experiments to observe hydrate formation and proposed that the formation of hydrates reduces the concentration of gas in the water. Additionally, Subramanian and Sloan [24] recorded Raman spectra just below the vapor/liquid interface during a continuous cooling process and qualitatively tracked the concentration of methane in the bulk aqueous phase in the absence and presence of hydrates, as predicted by CSMGem. The Raman spectra indicated a decrease in methane concentration in the aqueous phase upon hydrate formation, thereby providing direct evidence of an aqueous phase supply of methane for hydrate growth [24]

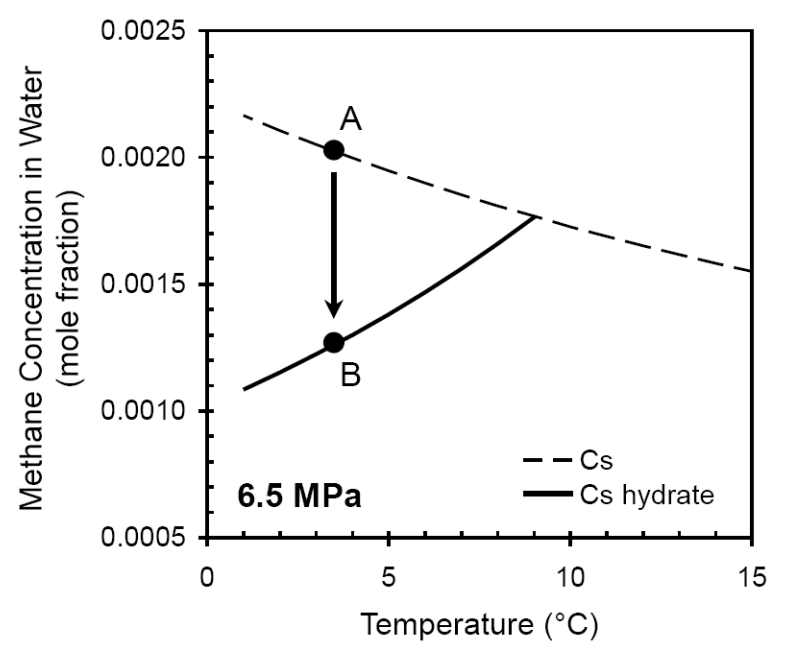

Figure 13. Methane solubility in the absence (dotted line) and presence (solid line) of hydrate (predicted using CSMGem).

\section{Growth Rates}

As mentioned previously, Freer et al. [12] studied the growth rate of methane hydrate propagating along the methane/water interface, establishing that hydrate growth rate increased with subcooling, as shown in Figure 14. Makogon et al. [8] also measured the growth rate of methane hydrate films at the free gas-water interface. He found that the growth rate was a function of both system pressure and degree of subcooling. For comparison, data collected from the current film growth measurements were plotted with the work by Freer et al. and Makogon et al., showing reasonable agreement between the three sets of measurements. 


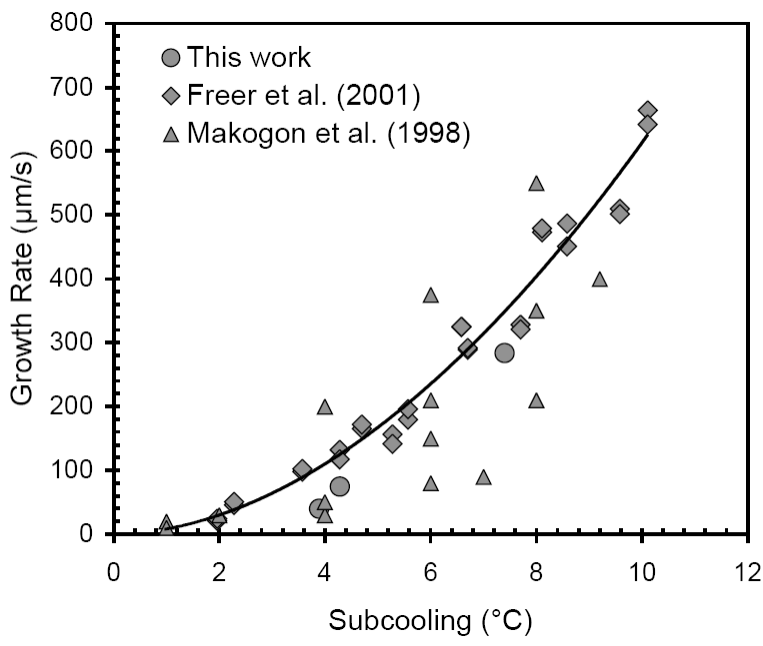

Figure 14. Methane hydrate film growth rate as a function of subcooling. Line represents power law trend through all data.

Although the growth rate with subcooling exhibited a convincing trend, solubility difference (mole fraction methane) in the absence and presence of hydrates can also be considered as a driving force for the hydrate growth rate. Figure 15 shows the growth rate as a function of solubility difference calculated by CSMGem [21] for the data reported in Figure 14. The greater driving force created a larger supersaturation of $\mathrm{CH} 4$ molecules in the liquid to sustain hydrate growth and thus a faster hydrate growth rate.

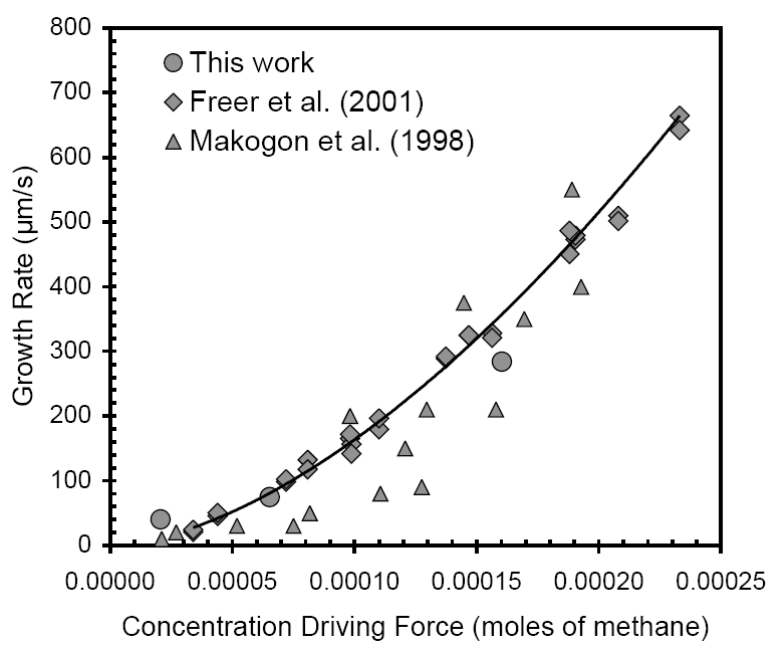

Figure 15. Methane hydrate growth rate as a function of concentration driving force. Line represents power law trend through all data.
Additionally, the concentration driving force may better characterize hydrate film thickness. The hydrate film thickness for methane hydrates is plotted against concentration driving force in Figure 16. The greater driving force created a larger supersaturation of $\mathrm{CH} 4$ molecules in the liquid to increase hydrate growth and thus a thicker hydrate film.

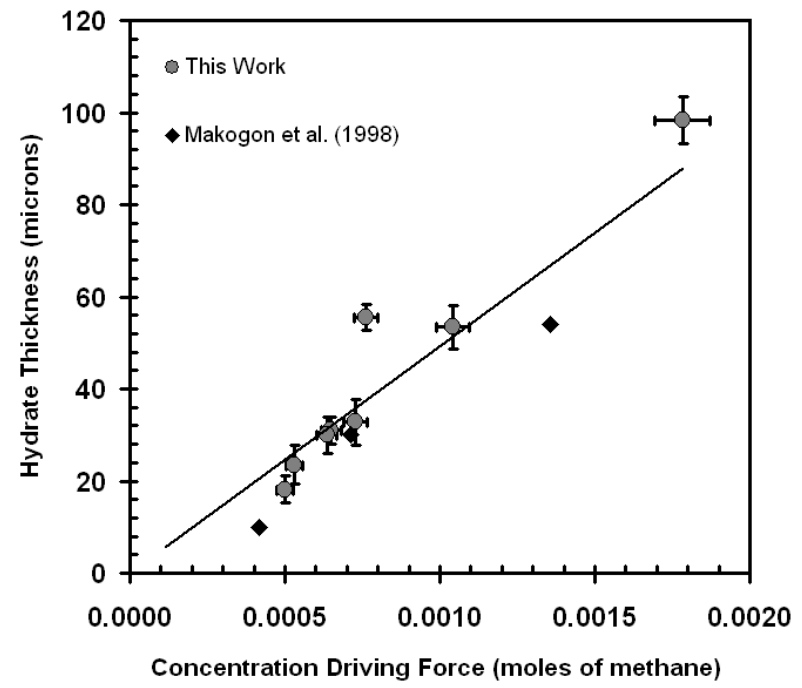

Figure 16. Methane hydrate film thickness as a function of concentration driving force. Line represents linear relationship trend through the all data.

\section{Proposed Mechanism of Hydrate Film Growth at the Hydrocarbon / Water Interface}

Hydrate film formation at the hydrocarbon/water interface was proposed to consist of three consecutive stages as illustrated in Figure 17. The first stage is the hydrate film formation, where a thin porous hydrate film propagates across the interface. The second stage is film development, in which the film thickens over time and pores within the hydrate film are filled. The third stage is bulk conversion of the hydrate film, in which all the remaining pores in the hydrate film are filled over a long period of time. 


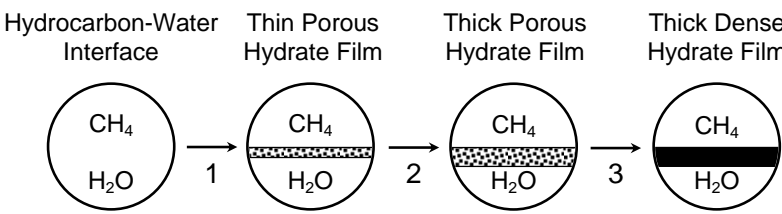

Figure 17. Schematic of the proposed mechanism for hydrate film formation at a hydrocarbon/water interface. Step 1: Propagation of a thin porous hydrate film across the hydrocarbon/water interface. Step 2: Film development. Step 3: Bulk conversion of hydrate film.

This proposed mechanism can also be applied to the conversion of a water droplet into a hydrate particle as illustrated in Figure 18. In a pipeline, a water droplet can be heterogeneously nucleated by a pre-existing hydrate particle. The first stage is the hydrate film formation, where a thin porous hydrate film propagates around the water droplet. The second stage is film development, in which the film thickens over time and some pores within the hydrate film are filled. The third stage is bulk conversion of the hydrate film, in which all the remaining pores in the hydrate film are filled and all the unconverted water on the interior of the hydrate is converted to hydrate over a long period of time.

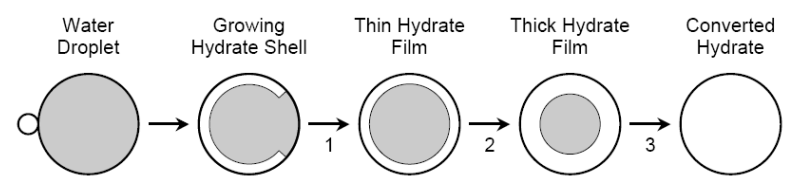

Figure 18. Schematic of the proposed mechanism for hydrate formation of a water droplet. Step 1: Propagation of a thin porous hydrate film around the water droplet. Step 2: Film development. Step 3: Bulk conversion of the hydrate.

While the film formation mechanism is quite general, in a pipeline the process will depend on thermodynamic conditions and type of hydrate structure formed (sI or sII). For methane hydrate, at $\sim 70$ bar ( 1000 psi) and $9.0^{\circ} \mathrm{C}$ of subcooling, the overall hydrate formation process takes place over one to two hours. In contrast, for cyclopentane hydrates at atmospheric pressure and $9.0{ }^{\circ} \mathrm{C}$ subcooling, the entire process takes over eight hours. A general time range for each step in the process is tens of seconds for the propagation step, tens of minutes for film development, and tens of hours for bulk conversion.
Transferability between Hydrate Formation for a Pool of Liquid Versus a Water Droplet

The transferability of the initial hydrate film thickness between a pool of water and a water droplet should be investigated. An initial methane hydrate film thickness of $\sim 5 \mu \mathrm{m}$ was measured for a planar water surface; however, the methane hydrate film thickness on a water droplet may be considerably different due to a greater surface area per unit volume of a droplet compared to a planar water surface. If the methane source for the initial shell growth is the aqueous phase (as suggested by the current experiments), the total moles of methane dissolved in the aqueous phase would be much less in a water droplet than in a pool of water. Therefore, significantly fewer moles of methane would be supplied from the aqueous phase to the hydrate film for a water droplet compared to a pool of water, perhaps resulting in a smaller initial hydrate film thickness.

\section{SUMMARY}

The hydrate film propagation rate across the hydrocarbon/water interface and the hydrate film thickness as a function of time were measured for methane and cyclopentane hydrate. Hydrate film thickness was directly proportional to subcooling and differed between methane and cyclopentane hydrate. Methane hydrate film thickness began at $\sim 5 \mu \mathrm{m}$ and grew to a final thickness (20 to 100 $\mu \mathrm{m})$ as a function of subcooling. The cyclopentane hydrate film thickness began at $\sim 12 \mu \mathrm{m}$ and grew to a final thickness (15 to $40 \mu \mathrm{m})$ as a function of subcooling. Gas consumption data during hydrate formation presented evidence of an aqueous phase supply of hydrate former to the initial hydrate growth, followed by a vapor phase supply of hydrate former in the thickening stage of hydrate film formation.

\section{REFERENCES}

[1] Sloan ED, Jr. Clathrate Hydrates of Natural Gases, second edition. Marcel Dekker, NY. 1998.

[2] Genov G, Kuhs WF, Staykova DK, Goreshnik $\mathrm{E}$, Slamatin AN, Experimental studies on the formation of porous gas hydrates. American Mineralogist 2004; 89:1228-1239.

[3] Mori Y.H. Estimating the thickness of hydrate films from their lateral growth rates: Application 
of a simplified heat transfer model. Journal of Crystal Growth 2001; 223: 206-212.

[4] Turner D.J. Clathrate hydrate formation in water-in-oil dispersions. Thesis, Colorado School of Mines, Golden, CO, 2005.

[5] Hirai S, Sanda H, Growth-controlling processes of $\mathrm{CO}_{2}$ gas hydrates. American Mineralogist 2004; 89: 1260-1263.

[6] Hirai S, Tabe Y, Kuwano K, Olgawa K, Okazaki K, MRI measurement of hydrate growth and application to advanced $\mathrm{CO}_{2}$ sequestration technology. Annals of the New York Academy of Sciences 2000b; 912: 246-253.

[7] Ito Y, Kamakura R., Obi S, Mori YH, Microscopic observations of clathrate-hydrate films formed at liquid/liquid interfaces. II. Film thickness in steady-water flow. Chemical Engineering Science 2003; 58:107-114.

[8] Makogon Y, Makogon T, Holditch S, Several aspects of the kinetics and morphology of gas hydrates, in: Proceedings of the Japan National Oil Conference 1998, pp. 259-267.

[9] Ohmura R, Kashiwazaki S, Mori YH, Measurements of clathrate-hydrate film thickness using laser interferometry. Journal of Crystal Growth 2000; 218: 372-380.

[10] Sugaya M, Mori YH, Behavior of clathrate hydrate formation at the boundary of liquid water and a fluorocarbon in liquid or vapor state. Chemical Engineering Science 1996;51:35053517.

[11] Uchida T, Ebinuma T, Narita $H$, Someya $S$, Interferometric observations of $\mathrm{CO}_{2}$ hydrate formation and growth, in: Proceedings of the Second International Symposium on Ocean Sequestration of Carbon Dioxide 1999b; Tokyo, pp. 1-6.

[12] Freer EM, Selim, MS, Sloan, ED Jr, Methane hydrate film growth kinetics. Fluid Phase Equilibria 2001; 185:65-75.

[13] Hirai S, Tabe Y, Kamijo S, Okazaki K, Propagation velocity of $\mathrm{CO}_{2}$ clathrate-hydrate film, in: Riemer P, Eliasson B, \& Wokaun A, editors. Greenhouse gas control technologies. Elsevier Science Ltd, 1999. p. 1049.

[14] Uchida T, Ikeda IY, Takeya S, Ebinuma T, Nagao J, Narita $\mathrm{H}, \mathrm{CO}_{2}$ hydrate film formation at the boundary between $\mathrm{CO}_{2}$ and water: Effects of temperature, pressure and additives on the formation rate. Journal of Crystal Growth 2002; 237-239: 383-387.

[15] Hirai S, Tabe Y, Kamijo S, Okazaki K, Lateral growth rates of $\mathrm{CO}_{2}$ clathrate-hydrate film. Thermal Science and Engineering 2000a; 8: $1-6$.

[16] Uchida T, Ebinuma T, Kawabata J, Narita H, Microscopic observations of formation processes of clathrate-hydrate films at an interface between water and carbon dioxide. Journal of Crystal Growth 1999a; 204: 348-356.

[17] Taylor, CJ. Adhesion force between hydrate particles and macroscopic investigation of hydrate film growth at the hydrocarbon/water interface. Thesis, Colorado School of Mines, Golden, CO, 2006.

[18] Servio P, Englezos P. A morphology study of methane and carbon dioxide gas hydrates formed from water droplets exposed to a gas atmosphere, in: Proceedings of the Fourth International Conference on Gas Hydrates. Yokohama, 2002, pp. $756-760$.

[19] Turner DJ, Kleehammer D, Koh C, Miller KT, Sloan ED Jr. Formation of Hydrate Obstructions in Pipelines Hydrate Particle Development and Slurry Flow, in: Proceedings of the Fifth International Conference on Gas Hydrates. Trondheim, 2005, pp. 1116-1125.

[20] Rasband WS. ImageJ. U.S. National Institutes of Health, Bethesda, MD. 2006. See also http://rsb.info.nih.gov/ij/

[21] Ballard AL, Sloan ED Jr. The next generation of hydrate prediction: An overview. Journal of Supramolecular Chemistry 2003; 2: 385-392.

[22] Tohidi B, Anderson R, Clennell B, Yang J, Bashir A, Burgass R. (). Application of high pressure glass micromodels to gas hydrates studies, in: Proceedings of the Fourth International Conference on Gas Hydrates. Yokohama, 2002, pp. 761-765.

[23] Tohidi B, Anderson R, Clennell MB, Burgass RW, Biderkab AB. Visual observation of gashydrate formation and dissociation in synthetic porous media by means of glass micromodels. Geology 2001; 29: 867-870.

[24] Subramanian S, Sloan, ED Jr. Solubility effects on growth and dissolution of methane hydrate needles, in: Proceedings of the Fourth International Conference on Gas Hydrates. Yokohama, 2002, pp. 856-861.

[25] Handa YP. Effect of hydrostatic pressure and salinity on the stability of gas hydrates. Journal of Physical Chemistry 1990; 94: 2652-2657. 